\title{
Design of Testing Period for Reliability Assessment of Numerical Control Machine Tools Considering Working Conditions
}

\author{
Li Hongzhou $\mathbb{D}^{1}$ and Sun Lixia $\mathbb{D}^{1,2}$ \\ ${ }^{1}$ College of Mechanical Engineering, Beihua University, Jilin 132013, China \\ ${ }^{2}$ College of Mechanical and Electric Engineering, Changchun University of Science and Technology, Changchun 130025, China \\ Correspondence should be addressed to Sun Lixia; sunlixiajilin@sohu.com
}

Received 10 March 2018; Revised 3 June 2018; Accepted 14 June 2018; Published 8 July 2018

Academic Editor: Elena Zaitseva

Copyright ( 2018 Li Hongzhou and Sun Lixia. This is an open access article distributed under the Creative Commons Attribution License, which permits unrestricted use, distribution, and reproduction in any medium, provided the original work is properly cited.

\begin{abstract}
The existing methods of determining testing period do not consider the effect of working condition covariates on reliability testing period of Numerical Control (NC) machine tools, which may lead to high-cost testing or low-precision assessment. Aiming at the problem, a new method of determining the testing period considering working condition covariates is proposed. The change rate of interval estimation of Mean Time between Failures (MTBF) is used as the criterion for determining the length of testing period. The reliability model of NC machine tools is established by the Cox proportional hazards model, and the two-step estimation method is used to estimate parameters of the baseline failure rate function and the coefficients of working condition covariates. The Bootstrap resamples are obtained by the Bootstrap resampling method. And then the parameters of the baseline failure rate function and the coefficients of working condition covariates are estimated simultaneously by maximum likelihood method, and thus interval estimations of MTBF under each covariate are obtained. The change rate models of MTBF interval estimation under each covariate level are established by Power function, and the testing periods under each covariate are obtained. Case study indicates that the testing periods under each covariate obtained by the proposed method are more accurate than those obtained by the others, when the same criterion and confidence level $1-\alpha$ are set.
\end{abstract}

\section{Introduction}

Field reliability test is the basic method of reliability assessment for NC machine tools. Unreasonable test period may decrease the assessment accuracy or increase the testing cost. In the premise of meeting evaluation accuracy, the shorter test period is, the lower test cost is. Therefore, it is of great practical engineering significance to choose an accurate test period [1]. Mchane determined the number of test pieces and test period based on the width of interval estimation of pivot, when Time between Failures (TBF) of the product followed logarithmic position-scale model [2]. He determined the test sample size by using the shortest interval estimation length of the evaluation parameter as the judgment basis [3]. Guo determined reliability test period by calculating the logarithmic ratio of upper interval estimation to lower interval estimation [4]. It can be learned from the above that the interval estimation accuracy of model parameters is usually taken as a criterion to determine the product's test period. In order to determine the suitable testing period for reliability assessment of NC machine tools, Li established the method of determining the testing period for reliability assessment of NC machine tools by using change rate of interval estimation as the criterion to determine the testing period [1]. However, influences of working conditions on the testing period were not considered in [1]. Guo calculated the front axle's fatigue life and obtained the enhancement coefficients of roads by comparative norm of tar-paved roads [5]. Chen adopted the multiple-stress accelerated test scheme to establish a reliability model of aerospace electrical connectors under different temperature and vibration stresses [6]. Qin studied the influence of the variation of environments and working conditions on the reliability of electronic system and proposed a system reliability evaluation method [7]. $\mathrm{Li}$ established the relationship between the reliability of harmonic driver and working conditions (including load and 
speed) by using the accelerated life test [8]. Ren presented a bathtub curve model, which included wear failure period and early failure period and analyzed the lasting time of early failure period of NC machine tools with minimal repair [9]. Zhou implemented the reliability assessment process of abrasion units and overloading units using Bayesian methods [10]. It can be deduced from the above studies that the worse the working conditions are, the shorter the testing period is. In other words, the better the working conditions are, the longer the test period is. Learning from [11], the scale parameter $\eta$ and MTBFs under the different covariate levels are different. And the more different the covariate level is, the more different the scale parameter $\eta$ and MTBFs are. Consequently, in determining the reliability test period of $\mathrm{NC}$ machine tools, not only the historical failure data but also the corresponding working conditions should be considered. The ignorance of working conditions may lead to inaccurate test period in [1].

Aiming at the above problem, according to the method in [1], the change rate of MTBF interval estimation of NC machine tools is also used as criterion to determine the testing period. Based on the historical failure data and the corresponding working condition of the NC machine tools, the reliability model of $\mathrm{NC}$ machine tools is obtained by the Cox proportional hazards model. The point estimations of the model's parameters are obtained by the two-step estimation method. The Bootstrap resamples are obtained by the Bootstrap resampling method. The parameters of the baseline failure rate function and the coefficients of working condition covariates are estimated simultaneously by maximum likelihood method, and then interval estimations of MTBF under each covariate are obtained. The change rate models of MTBF interval estimation under each covariate level are established by Power function, and the testing periods under each covariate are obtained. Finally, the case study indicates the proposed method is feasible.

\section{Reliability Model of NC Machine Tools Considering Working Conditions}

For convenience, the failure rate function of NC machine tools under working condition $\mathrm{Z}$ in [11] is rewritten as

$$
\lambda\left(\frac{t}{Z}\right)=\frac{m}{\eta}\left(\frac{t}{\eta}\right)^{m-1} \cdot \exp \left[\beta\left(Z-Z_{1}\right)\right]
$$

where $Z$ is a vector of working condition covariates (for example, cutting force, number of tool changes, and vibration) which affects the failure rate of NC machine tools and $Z_{1}$ is the baseline working condition covariate which is selected from $Z$. $\beta=\left(\beta_{1}, \beta_{2}, \cdots, \beta_{k}\right)$ is the vector of $Z$ 's coefficients, which reflects the covariates' influences on the failure rate function. $m$ is the shape parameter under $Z_{1}$ and $m>0 ; \eta$ is the scale parameter under $Z_{1}$ and $\eta>0$.

Then the reliability function of NC machine tools under working conditions $Z$ can be written as follows:

$$
R\left(\frac{t}{Z}\right)=\left\{\exp \left[-\left(\frac{t}{\eta}\right)^{m}\right]\right\}^{\exp \left[\beta\left(Z-Z_{1}\right)\right]}
$$

Then, the probability distribution function (PDF) of NC machine tools under working conditions $Z$ can be written as follows:

$$
\begin{aligned}
f\left(\frac{t}{Z}\right)= & \left\{\exp \left[-\left(\frac{t}{\eta}\right)^{m}\right]\right\}^{\exp \left[\beta\left(Z-Z_{1}\right)\right]} \cdot \frac{m}{\eta}\left(\frac{t}{\eta}\right)^{m-1} \\
& \cdot \exp \left[\beta\left(Z-Z_{1}\right)\right] .
\end{aligned}
$$

Then, the MTBF of NC machine tools under covariate $Z_{1}$ is obtained by

$$
M T B F=\eta \times \Gamma\left(1+\frac{1}{m}\right)
$$

where $\Gamma(1+1 / m)$ is Gamma function.

$\widehat{m}, \widehat{\eta}, \widehat{\eta}_{j}$, and $\widehat{\beta}$, which are the estimations of parameters $m, \eta, \eta_{j}$, and coefficients $\beta$, are obtained by the two-step estimation method in [11]. And the covariates which have no effects on the reliability of NC machine tools are eliminated by the likelihood ratio test in [11]. $\eta_{j}$, which is the scale parameter of NC machine tools under covariate $Z_{j}$, are obtained by (5) written in [11].

$$
\eta_{j}=\frac{\eta}{\exp \left[(\beta / m)\left(Z_{j}-Z_{1}\right)\right]}
$$

Then, $M T B F_{j}$ of NC machine tools under working conditions covariate $Z_{j}$ is expressed as

$$
M T B F_{j}=\eta_{j} \times \Gamma\left(1+\frac{1}{m}\right) .
$$

\section{Design of Reliability Testing Period}

Step 1. The historical failure data and the corresponding working conditions of the NC machine tools to be tested are collected and used to establish the reliability model of NC machine tools considering working conditions in (1). The point estimations $\widehat{m}, \widehat{\eta}, \widehat{\eta}_{j}$, and $\widehat{\beta}$ of parameters $m, \eta$, $\eta_{j}$, and coefficients $\beta$ of the reliability model are calculated by the two-step estimation method in [11]. And then the point estimations $\widehat{M T B F}_{j}$ of $M T B F_{j}$ of the NC machine tools under $Z_{j}$ are obtained by (6).

Step 2. A testing period $\mathrm{T}$ is set. A Bootstrap resampling is obtained by the Bootstrap resampling method [12] based on the point estimations $\widehat{m}, \widehat{\eta}, \widehat{\eta}_{j}$, and $\widehat{\beta}$ obtained in Step 1 .

Step 3. The working condition covariates in (1) that have no effects on the reliability of NC machine tools are eliminated by the two-step estimation method and the likelihood ratio test in [11]. Thus, the parameters $\widehat{m}, \widehat{\eta}, \widehat{\eta}_{j}$ and the coefficient $\widehat{\beta}$ are not calculated by the two-step estimation method but calculated simultaneously by the maximum likelihood method.

The maximum likelihood function of NC machine tools for the Bootstrap resampling sample is given as

$$
L(\eta, m)=\prod_{i=1}^{r} f\left(\frac{t_{i}}{Z_{i}}\right) \prod_{i=r+1}^{n} R\left(\frac{t_{i}}{Z_{i}}\right)
$$


where $r$ is the number of failure data. $n$ is the sum of failure data and timing censored data. $Z_{i}$ is the ith working condition covariate.

Substituting (2) and (3) into (7), the likelihood function is obtained as

$$
\begin{aligned}
L(\eta, m) & \prod_{i=1}^{r}\left\{\frac{m}{\eta}\left(\frac{t_{i}}{\eta}\right)^{m-1} \cdot \exp \left[\beta\left(Z_{i}-Z_{1}\right)\right]\right\} \\
& \cdot \prod_{i=1}^{n}\left\{\exp \left[-\left(\frac{t_{i}}{\eta}\right)^{m}\right]\right\}^{\exp \left[\beta\left(Z_{i}-Z_{1}\right)\right]} \\
= & m^{r} \eta^{-r m}\left(t_{1} t_{2} \cdots t_{r}\right)^{m-1} \\
& \cdot \exp \left\{-\left[\left(\frac{t_{1}}{\eta}\right)^{m}+\left(\frac{t_{2}}{\eta}\right)^{m}+\cdots+\left(\frac{t_{r}}{\eta}\right)^{m}\right]\right\} \\
& \cdot \exp \left[\beta\left(Z_{1}+Z_{2}+\cdots+Z_{r}-r Z_{1}\right)\right] \\
& \cdot \prod_{i=r+1}^{n} \exp \left[-\left(\frac{t_{i}}{\eta}\right)^{m} \exp \left[\beta\left(Z_{i}-Z_{1}\right)\right]\right] .
\end{aligned}
$$

Taking the logarithm of both sides in (8), then

$$
\begin{aligned}
\ln [L(\eta, m)]= & r \ln m-r m \ln \eta \\
& +(m-1) \ln \left(t_{1} t_{2} \cdots t_{r}\right) \\
& -\left[\left(\frac{t_{1}}{\eta}\right)^{m}+\left(\frac{t_{2}}{\eta}\right)^{m}+\cdots+\left(\frac{t_{r}}{\eta}\right)^{m}\right] \\
& +\beta\left(Z_{1}+Z_{2}+\cdots+Z_{r}-r Z_{1}\right) \\
& +\sum_{i=r+1}^{n}\left[-\left(\frac{t_{i}}{\eta}\right)^{m} \exp \left[\beta\left(Z_{i}-Z_{1}\right)\right]\right] .
\end{aligned}
$$

Taking the partial derivatives of the parameters $m, \eta$, and $\beta$ in (9), respectively, then

$$
\begin{aligned}
& \frac{\partial \ln [L(\eta, m)]}{\partial \eta}=-\frac{r m}{\eta}+\left[m t_{1}^{m} \eta^{(-m-1)}+m t_{2}^{m} \eta^{(-m-1)}\right. \\
& \left.+\cdots+m t_{r}^{m} \eta^{(-m-1)}\right] \\
& +\sum_{i=r+1}^{n}\left[m t_{i}^{m} \eta^{(-m-1)} \exp \left[\beta\left(Z_{i}-Z_{1}\right)\right]\right] \\
& \frac{\partial \ln [L(\eta, m)]}{\partial m}=\frac{r}{m}-r \ln \eta+\ln \left(t_{1} t_{2} \cdots t_{r}\right) \\
& -\left[\left(\frac{t_{1}}{\eta}\right)^{m} \ln \frac{t_{1}}{\eta}+\left(\frac{t_{2}}{\eta}\right)^{m} \ln \frac{t_{2}}{\eta}+\cdots\right. \\
& \left.+\left(\frac{t_{r}}{\eta}\right)^{m} \ln \frac{t_{r}}{\eta}\right]^{n}\left[-\left(\frac{t_{i}}{\eta}\right)^{m} \ln \left(\frac{t_{i}}{\eta}\right) \exp \left[\beta\left(Z_{i}-Z_{1}\right)\right]\right]
\end{aligned}
$$

$$
\begin{aligned}
& \frac{\partial \ln [L(\eta, m)]}{\partial \beta}=\left(Z_{1}+Z_{2}+\cdots+Z_{r}-r Z_{1}\right) \\
& +\sum_{i=r+1}^{n}\left[-\left(\frac{t_{i}}{\eta}\right)^{m} \cdot\left(Z_{i}-Z_{1}\right) \cdot \exp \left[\beta\left(Z_{i}-Z_{1}\right)\right]\right]
\end{aligned}
$$

Because (10), (11), and (12) have no analytical solutions, Newton-Raphson numerical algorithm is used to estimate parameters $\eta, m$, and $\beta$.

$M T B F_{Z_{1}}$ of the NC machine tools under the baseline working condition covariate $Z_{1}$ are obtained by (4).

$\eta_{i}$ of the NC machine tools under the working condition covariates $Z_{i}$ are obtained by (5) and rewritten as

$$
\eta_{i}=\frac{\eta}{\exp \left[(\beta / m)\left(Z_{i}-Z_{1}\right)\right]}
$$

$M T B F_{Z_{i}}$ of the NC machine tools under the working condition covariate $Z_{i}(i=2,3, \cdots)$ are obtained by (6) and rewritten as

$$
\operatorname{MTBF}_{\mathrm{Z}_{i}}=\eta_{i} \times \Gamma\left(1+\frac{1}{m}\right), \quad i=2,3, \ldots
$$

Step 4. B resampling samples are obtained by Bootstrap resampling method based on $\widehat{m}, \widehat{\eta}, \widehat{\eta}_{j}, \widehat{\beta}$ which are obtained in Step 1. The point estimation of MTBF under $Z_{i}$ for each Bootstrap resampling are calculated by Step 2 to Step 3 and expressed as $M T B F_{Z_{i}}^{b}, b=1,2, \cdots, B, i=1,2, \cdots, q$.

Step 5. $M T B F_{Z_{i}}^{b}, b=1,2, \cdots, B$, under covariate $Z_{i}$ obtained by Step 4 are arranged in ascending order, and then $q$ new sequences are gotten as follows:

$$
\begin{array}{cl}
\operatorname{MTBF}_{\mathrm{Z}_{1}}^{b^{\prime}}, & b^{\prime}=1,2, \ldots, B \\
\operatorname{MTBF}_{\mathrm{Z}_{2}}^{b^{\prime}}, & b^{\prime}=1,2, \ldots, B \\
\vdots & \\
\operatorname{MTBF}_{\mathrm{Zq}}^{b^{\prime}}, & b^{\prime}=1,2, \ldots, B .
\end{array}
$$

Step 6. Set confidence level 1- $\alpha$. Solve and round $(B \cdot(\alpha / 2))$ and $(B \cdot(1-\alpha / 2)$, respectively. Then two quantiles $(B \cdot(\alpha / 2))$ and $(B \cdot(1-\alpha / 2)$ of each of the $\mathrm{q}$ sequences in Step 5 are selected as the upper limit and lower limit of $M T B F$ interval estimation under covariate $Z_{i}$. The $M T B F$ interval estimations of NC machine under covariate $Z_{i}$ are expressed as

$$
\begin{gathered}
{\left[\operatorname{MTBF}_{\mathrm{Z}_{1}}^{(B \cdot(\alpha / 2))}, \operatorname{MTBF}_{\mathrm{Z}_{1}}^{(B \cdot(1-\alpha / 2))}\right]} \\
{\left[\operatorname{MTBF}_{\mathrm{Z}_{2}}^{(B \cdot(\alpha / 2))}, \operatorname{MTBF}_{\mathrm{Z}_{2}}^{(B \cdot(1-\alpha / 2))}\right]} \\
\vdots \\
{\left[\operatorname{MTBF}_{\mathrm{Z}_{q}^{(B \cdot(\alpha / 2))},}, \operatorname{MTBF}_{\mathrm{Z}_{q}}^{(B \cdot(1-\alpha / 2))}\right] .}
\end{gathered}
$$


Step 7. Set $l$ testing periods. $T_{1}<T_{2}<\cdots<T_{l}$. The MTBF interval estimations of NC machine tools under covariate $Z_{i}$ for each testing period $T_{h}(h=1,2, \cdots, l)$ are obtained by Step 2 to Step 6 and expressed as

$$
\begin{gathered}
{\left[\operatorname{MTBF}_{\mathrm{Z}_{1}, T_{h}}^{(B \cdot(\alpha / 2))}, \operatorname{MTBF}_{\mathrm{Z}_{1}, T_{h}}^{(B \cdot(1-\alpha / 2))}\right]} \\
{\left[\operatorname{MTBF}_{\mathrm{Z}_{2}, T_{h}}^{(B \cdot(\alpha / 2))}, \operatorname{MTBF}_{\mathrm{Z}_{2}, T_{h}}^{(B \cdot(1-\alpha / 2))}\right]} \\
\vdots \\
{\left[\operatorname{MTBF}_{\mathrm{Z}_{q}, T_{h}}^{(B \cdot(\alpha / 2))}, \operatorname{MTBF}_{\mathrm{Z}_{q}, T_{h}}^{(B \cdot(1-\alpha / 2))}\right]} \\
h=1,2,3, \ldots, l .
\end{gathered}
$$

Step 8. q curves, which are to be fitted, respectively,

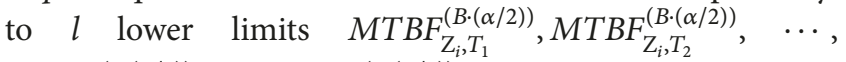
$\operatorname{MTBF}_{\mathrm{Z}_{i}, T_{h}}^{(B \cdot(\alpha / 2))}, \cdots, \operatorname{MTBF}_{\mathrm{Z}_{i}, T_{l}}^{(B \cdot(\alpha))}(i=1,2,3, \cdots, q)$, are obtained by Matlab Cftool and expressed as

$$
H_{l o w}^{Z_{i}}(T), \quad i=1,2,3, \ldots, q,
$$

where $Z_{i}$ is the ith working condition covariate. Low is the lower limits of interval estimations. $H_{\text {low }}^{Z_{i}}(T)$ is the curve fitting function of lower limits under working condition covariate $Z_{i}$.

In the same way, the curves, which are to be fitted, respectively, to the upper limits of $l \mathrm{MTBF}$ interval estimations, $\operatorname{MTBF}_{\mathrm{Z}_{i}, T_{1}}^{(B \cdot(1-\alpha / 2))}, \operatorname{MTBF}_{\mathrm{Z}_{i}, T_{2}}^{(B \cdot(1-\alpha / 2))}, \cdots, \operatorname{MTBF}_{\mathrm{Z}_{i}, T_{h}}^{(B \cdot(1-\alpha / 2))}$, $\cdots, \operatorname{MTBF}_{\mathrm{Z}_{i}, T_{l}}^{(B \cdot(-\alpha / 2))}(i=1,2,3, \cdots, q)$, are also obtained by Matlab Cftool and expressed as

$$
H_{u p}^{Z_{i}}(T), \quad i=1,2,3, \ldots, q,
$$

where $Z_{i}$ is the ith working condition covariate. Up is the upper limits of interval estimations. $H_{u p}^{Z_{i}}(T)$ is the curve fitting function of upper limits under working condition covariate $Z_{i}$.

And then, the fitting functions of $M T B F$ interval estimations of NC machine tools under covariate $Z_{i}$ can be obtained from (18) to (19) and expressed as

$$
F^{Z_{i}}(T)=H_{u p}^{Z_{i}}(T)-H_{\text {low }}^{Z_{i}}(T), \quad i=1,2,3, \ldots, q,
$$

where the variables are the same as those of (18) and (19).

Step 9. Change rates of $M T B F$ interval estimation under covariate $Z_{i}$ are obtained by taking the derivative of (20) and expressed

$$
\begin{array}{r}
Q^{Z_{i}}(T)=\frac{\partial F^{Z_{i}}(T)}{\partial T}=\frac{\partial H_{u p}^{Z_{i}}(T)}{\partial T}-\frac{\partial H_{l o w}^{Z_{i}}(T)}{\partial T}, \\
i=1,2,3, \ldots, q,
\end{array}
$$

where the variables are the same as those of (20).

Learning from [1], $Q^{Z_{i}}(T)$ are also taken as the criterion that determines how long testing period of reliability assessment is. Suppose $Q_{M T B F}$ is the change rate of MTBF interval
TABLE 1: Reliability parameters of the NC machine tools under each covariate.

\begin{tabular}{lcccc}
\hline \multicolumn{2}{c}{ Covariates } & Shape & Scale & MTBF \\
$F c(\mathrm{KN})$ & $N h(\mathrm{n} / \mathrm{h})$ & & & \\
\hline 0.35 & 2 & 1.2121 & 1156 & 1084.6 \\
0.43 & 17 & 1.2121 & 584.5916 & 548.5002 \\
0.54 & 4 & 1.2121 & 891.4274 & 836.4116 \\
0.78 & 10 & 1.2121 & 557.8750 & 523.4331 \\
0.81 & 4 & 1.2121 & 691.2005 & 648.5274 \\
0.84 & 6 & 1.2121 & 619.7461 & 581.4844 \\
1.03 & 14 & 1.2121 & 374.9816 & 351.8384 \\
\multicolumn{7}{l}{ The method in $[1]$} & 1.1468 & 644.8311 & 614.1914 \\
\hline
\end{tabular}

estimation required before the reliability assessment test; then the testing period $T$ under covariate $Z_{i}$ must be determined by

$$
\left|Q^{Z_{i}}(T)\right|=\left|\frac{\partial F(T)}{\partial T}\right| \leq Q_{M T B F} \quad i=1,2,3, \ldots, q .
$$

Step 10. In order to test (18) and (19), tests of goodness-of-fit are made [13].

Suppose $y$ referred to as the sample value is upper limit or lower limit of MTBF interval estimation obtained by the Bootstrap resampling. $\bar{y}$ is the average of $y$. Suppose $\widehat{y}$ referred to as theoretical value of fitting is the upper limit or lower limit of MTBF interval estimation obtained by the curves fitting. The error quadratic sum of $\hat{y}$ is expressed as

$$
S S_{c}=\sum(y-\widehat{y})^{2} \text {. }
$$

The mean square error of $\hat{y}$ is expressed as

$$
S S_{z}=\sum(y-\bar{y})^{2} \text {. }
$$

The $\mathrm{R}$ square is calculated by

$$
R^{2}=1-\frac{S S_{c}}{\mathrm{SS}_{z}}=1-\frac{\sum(y-\widehat{y})^{2}}{\sum(y-\bar{y})^{2}}
$$

where $R^{2}$ represents the degree of nonlinear correlation between $y$ and $\hat{y}$. The range of $R^{2}$ is $[0,1]$. When $R^{2}$ is closer to 1 , the nonlinear correlation is better. when $R^{2}$ is closer to 0 , the nonlinear correlation is worse.

\section{Case Study}

Suppose the NC machine tools in [11] have been improved by the manufacturer several years later, and the reliability of the improved NC machine tool will be evaluated. Thus, the testing data of NC machine in [11] can be regarded as the history fault information of the improved NC machine tools, which are used to determine the reliability testing period of the improved NC machine tools before the test by the proposed method.

The point estimations of reliability parameters (such as shape, scale, and MTBF) of the NC machine tools under each covariate are obtained by Step 1 and listed in Table 1 . And for 


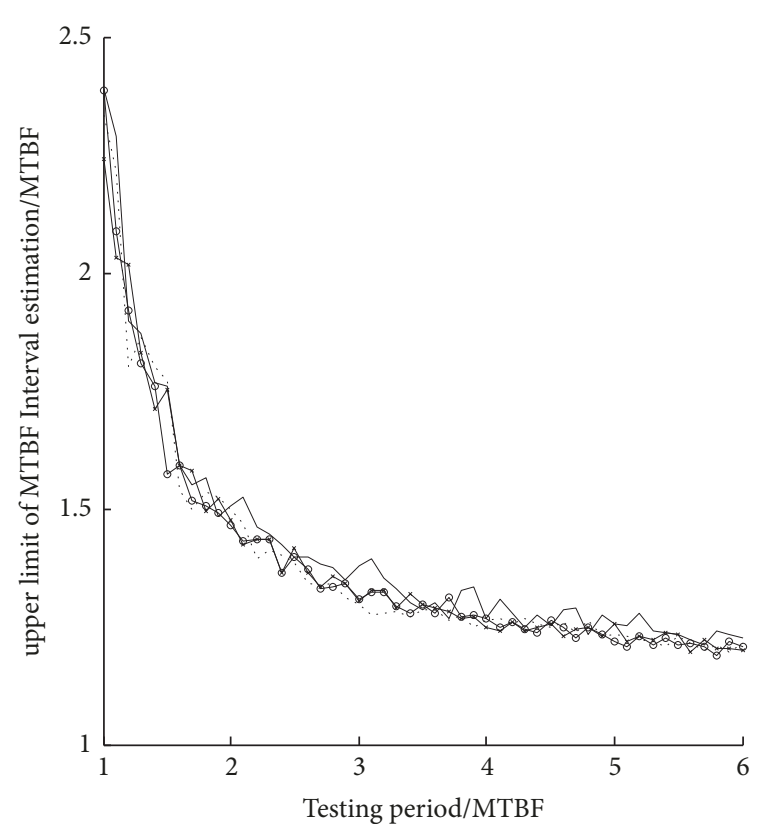

$$
\ldots . \mathrm{Fc}=0.35, \mathrm{Nh}=2 \quad \longrightarrow \mathrm{Fc}=0.54, \mathrm{Nh}=4
$$

(a) Upper limit of interval estimations of MTBF under different covariate

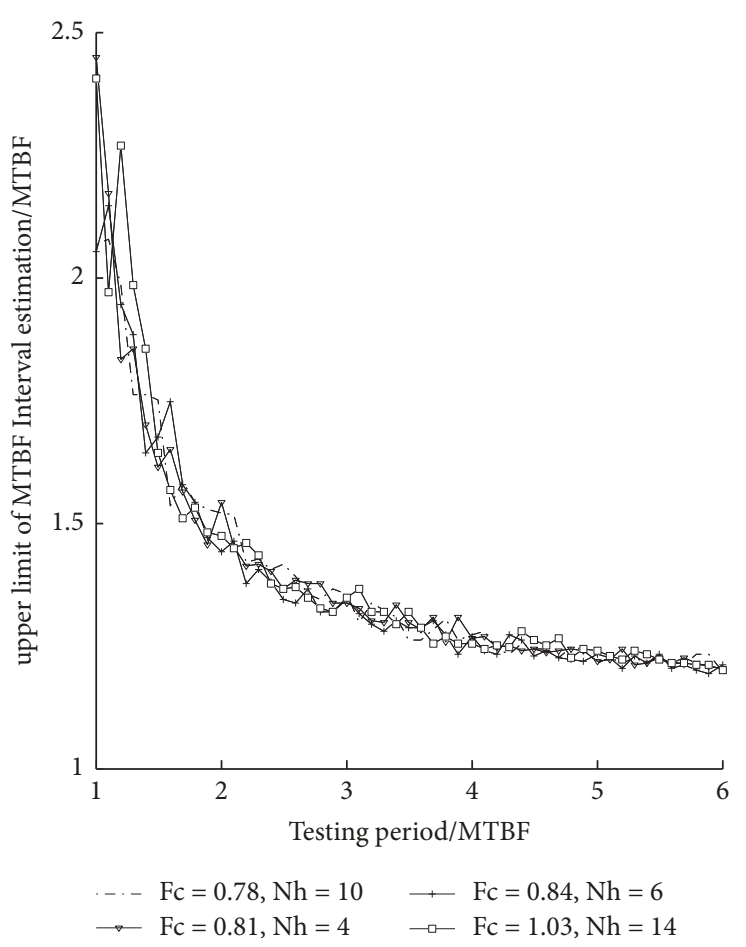

(b) Upper limit of interval estimations of MTBF under different covariate

FiguRe 1

TABLE 2: The curves fitted to the upper limit or the lower limit of MTBF interval estimation under different covariates.

\begin{tabular}{|c|c|c|c|c|c|}
\hline \multicolumn{2}{|c|}{ Covariates } & \multirow{2}{*}{$\begin{array}{l}\text { The curves fitted to the upper limit of } \\
\text { MTBF interval estimation }\end{array}$} & \multirow{2}{*}{$R^{2}$} & \multirow{2}{*}{$\begin{array}{l}\text { The curves fitted to the lower limit of } \\
\text { MTBF interval estimation }\end{array}$} & \multirow{2}{*}{$R^{2}$} \\
\hline$F c(\mathrm{KN})$ & $N h(\mathrm{n} / \mathrm{h})$ & & & & \\
\hline 0.35 & 2 & $1.202 * T^{-2.044}+1.19$ & 0.9567 & $-0.2866 * T^{-0.568}+0.9673$ & 0.9791 \\
\hline 0.43 & 17 & $1.026 * T^{-1.627}+1.147$ & 0.9711 & $-0.2642 * T^{-0.6123}+0.9501$ & 0.9869 \\
\hline 0.54 & 4 & $1.152 * T^{-2.223}+1.2121$ & 0.9785 & $-0.2727 * T^{-0.6218}+0.9517$ & 0.9813 \\
\hline 0.78 & 10 & $1.004 * T^{-1.467}+1.135$ & 0.9804 & $-0.2528 * T^{-0.7512}+0.9277$ & 0.9835 \\
\hline 0.81 & 4 & $1.066 * T^{-1.798}+1.173$ & 0.9895 & $-0.2549 * T^{-0.6905}+0.937$ & 0.9815 \\
\hline 0.84 & 6 & $1.116 * T^{-2.183}+1.204$ & 0.9879 & $-0.3049 * T^{-0.5086}+0.9872$ & 0.9830 \\
\hline 1.03 & 14 & $1.134 * T^{-2.05}+1.19$ & 0.9766 & $-0.283 * T^{-0.5508}+0.9688$ & 0.9667 \\
\hline \multicolumn{2}{|c|}{ The method in [1] } & $1.162 * T^{-2.084}+1.22$ & 0.9796 & $-0.2865 * T^{-0.6191}+0.9515$ & 0.9846 \\
\hline
\end{tabular}

comparison, the point estimations of reliability parameters (such as shape, scale, and MTBF) of the NC machine tools obtained by the method without considering covariates in [1] are also listed in Table 1.

Based on the point estimate of the reliability parameter in Table 1, the reliability test periods of the improved NC machine tool are designed by the proposed method in this paper. Then the upper limits and lower limits of MTBF interval estimations under covariate $Z_{i}$ of each Bootstrap resampling for testing period $\mathrm{T}$ are obtained by Step 2 to Step 7. The upper limits of interval estimations of MTBF under different covariate are shown in Figures 1(a) and 1(b), while the lower limits of interval estimations of MTBF under different covariate are shown in Figures 2(a) and 2(b). In order to facilitate comparison, the coordinates in Figures 1(a), 1(b), 2(a), and 2(b) are normalized. In other words, the lower limits or upper limits of MTBF interval estimations under each covariate are divided by the MTBF of NC machine tools under corresponding covariates in Figures 1(a), 1(b), 2(a), and 2(b). The upper limits and lower limits of MTBF interval estimations obtained by the method in [1] are also shown in Figures 1(a) and 2(a), respectively.

The curves fitted to the lower limits or upper limits of MTBF interval estimations under each covariate are calculated by Step 8 and shown in Table 2. The curves fitted to the lower limits or upper limits of MTBF interval estimations are obtained by the method in [1] and also shown in Table 2. $R^{2}$ are calculated by Step 10 and listed in Table 2 .

The change rates of $M T B F$ interval estimation of NC machine tools under covariate $Z_{i}$ are obtained by Step 9 based 


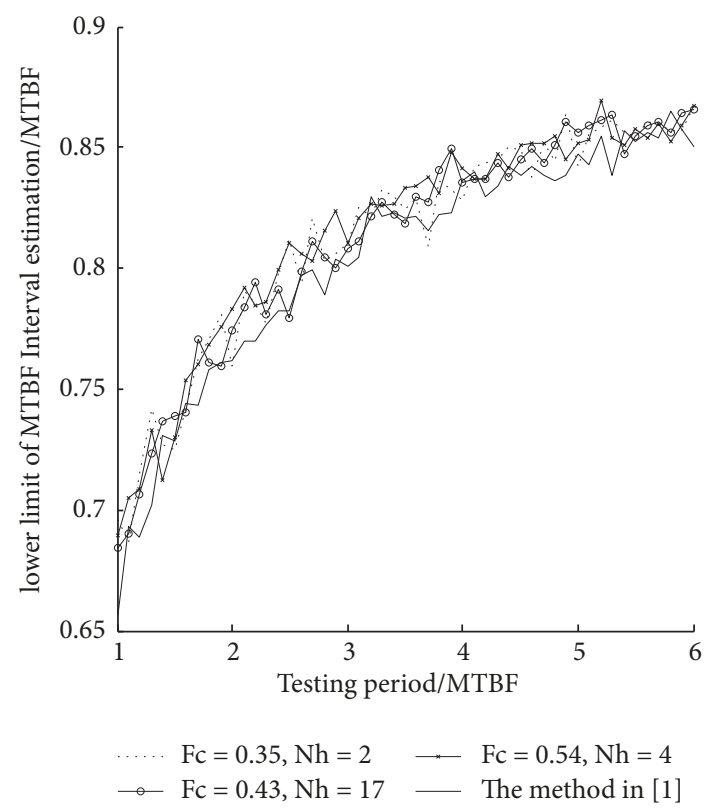

(a) Lower limit of interval estimations of MTBF under different covariate

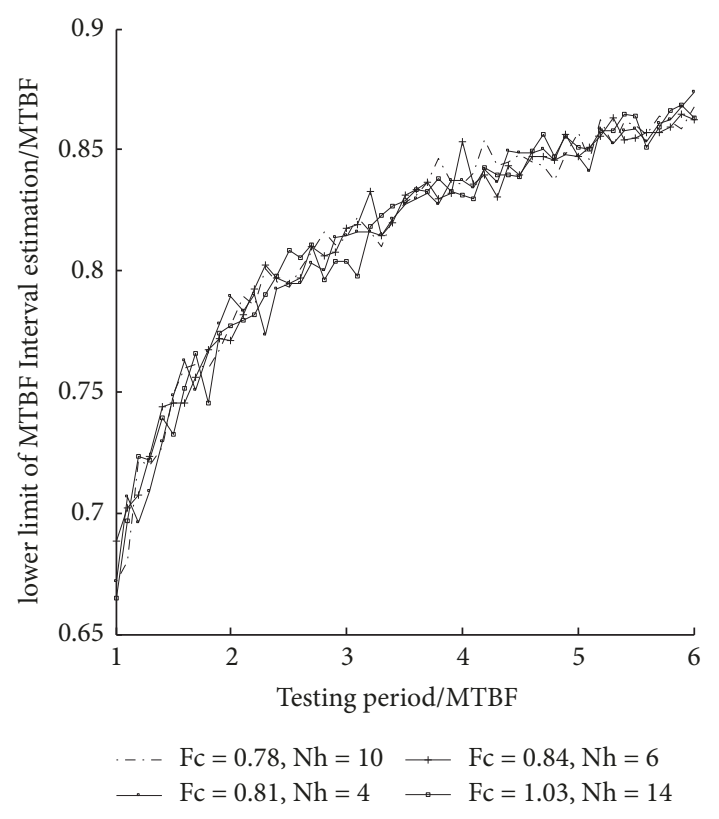

(b) Lower limit of interval estimations of MTBF under different covariate

Figure 2

TABLE 3: Testing periods of NC machine tools under different covariates.

\begin{tabular}{lccccc}
\hline \multicolumn{2}{c}{ Covariates } & \multicolumn{3}{c}{ Testing period } \\
$\begin{array}{l}F c \\
(\mathrm{KN})\end{array}$ & $\mathrm{Nh}$ & /MTBF & /h & MTBF difference & Change rate of MTBF interval estimation \\
\hline 0.35 & 2 & 1.5 & 1627 & 645 & -0.18 \\
0.43 & 17 & 1.6 & 878 & -104 & -0.192 \\
0.54 & 4 & 1.6 & 1338 & 356 & -0.185 \\
0.78 & 10 & 1.6 & 837 & -145 & -0.192 \\
0.81 & 4 & 1.5 & 973 & -9 & -0.193 \\
0.84 & 6 & 1.6 & 930 & -52 & -0.187 \\
1.03 & 14 & 1.6 & 563 & -419 & -0.19 \\
\multicolumn{2}{l}{ The method in $[1]$} & 1.6 & 982 & 0 (baseline) & -0.19 \\
\hline
\end{tabular}

on Table 2 and shown in Figures 3(a) and 3(b). The change rates of MTBF interval estimation of NC machine tools are also obtained by the method in [1] and shown in Figures 3(a) and 3(b). Figure 3(b) is the enlargement of $\mathrm{H}$ part in Figure 3(a).

The testing periods of $\mathrm{NC}$ machine tools under different covariates are listed in Table 3 and Figure 4. The testing period of NC machine tools obtained by the method in [1] is also listed in Table 3 and Figure 4. The fourth column in Table 3 is equal to the third column times the MTBF of the NC machine tools under the corresponding covariate. For comparison, the testing period of 982 hours obtained by the method in [1] is used as the benchmark; the fifth column is equal to the fourth column minus 982 hours.

Set $Q_{M T B F}=0.2$; learning from the third column in Table 3, the testing periods obtained by the proposed method and by the method in [1] are all between 1.5 times of MTBF and 1.6 times of MTBF, and their difference is very little.
However, when the unit of the test period of NC machine tools is converted from MTBF multiple to hour, the test period of NC machine tools will be significantly different, which is shown as the fourth column in Table 3. From the fourth column or fifth column in Table 3, when the testing period of 982 hours of NC machine tools obtained by the method in [1] is used as the benchmark, the testing periods of NC machine tools under some covariate level are very different from the benchmark. For example, when $\mathrm{Fc}=1.03$ and $\mathrm{Nh}=14$, the testing period of NC machine tools is 419 hours shorter than the benchmark. When $\mathrm{Fc}=0.35, \mathrm{Nh}=2$, the testing period of NC machine tools is 645 hours longer than the benchmark. When $\mathrm{Fc}=1.03, \mathrm{Nh}=14$, the testing period of NC machine tools is 1064 hours longer than that of NC machine tools under $\mathrm{Fc}=0.35, \mathrm{Nh}=2$. It can be deduced from the above analyses that the test periods obtained by the proposed method are quite different from the results obtained by the method in [1]. 


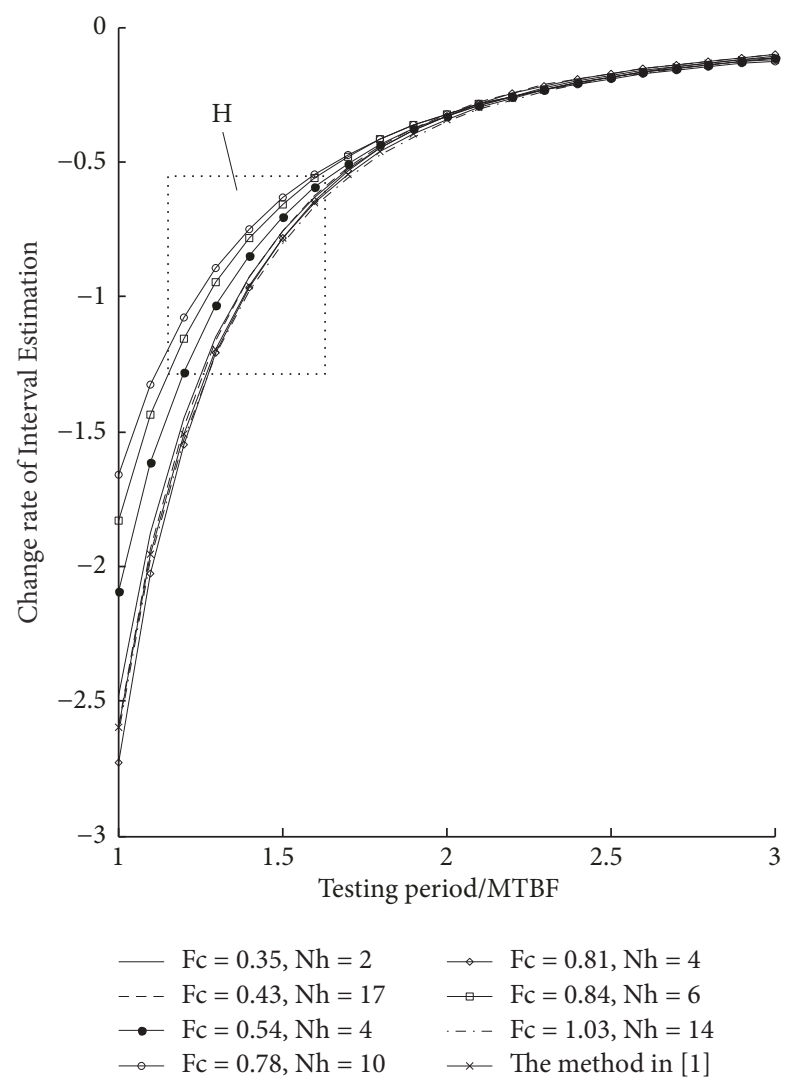

(a) The change rate of MTBF interval estimation under different covariates

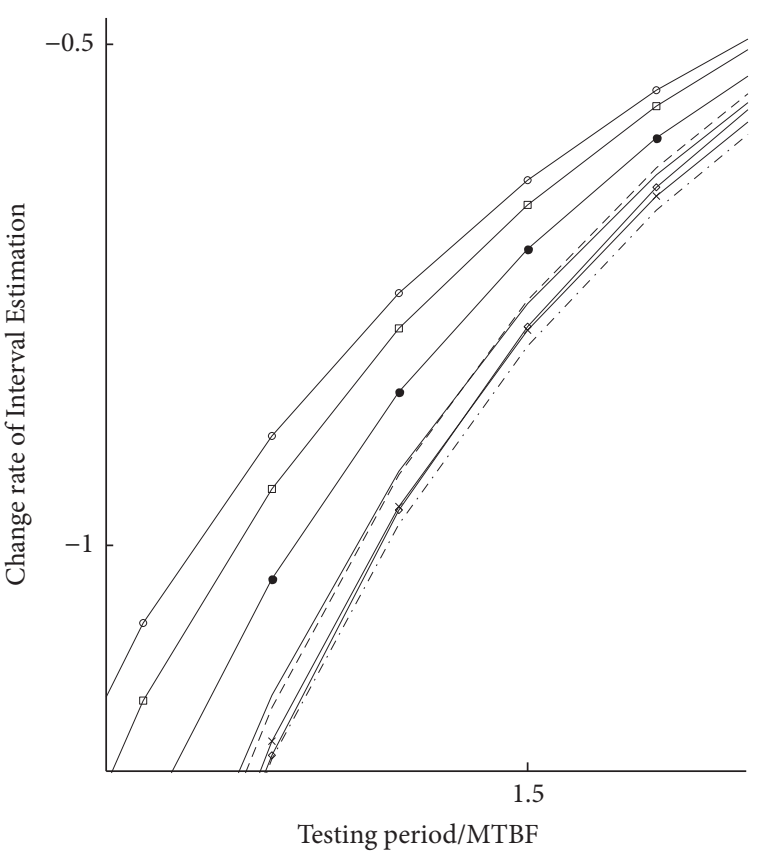

(b) Partial enlarged view of $\mathrm{H}$ part in Figure 3(a)

FigURE 3

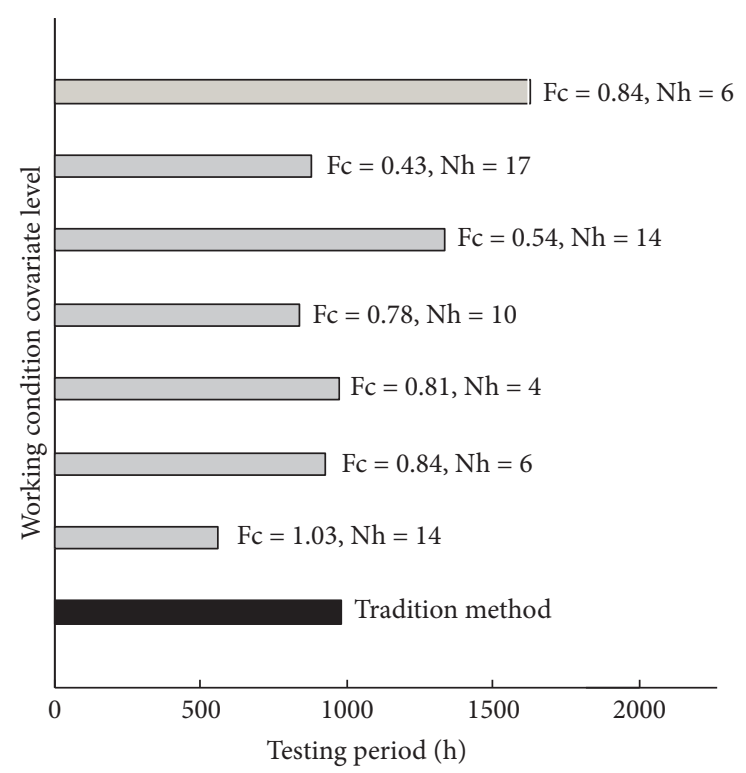

FIGURE 4: Testing period of NC machine tools under different covariates.

\section{Conclusions}

The same NC machine tools under different covariates may have different testing periods, when the same criterion for determining the testing period is set. However, the existing methods including that in [1] do not consider the impacts of working conditions covariates on the testing period. Considering this problem, the authors propose a new method to determine the testing period considering working conditions. The proportional hazards model is used to establish reliability 
model of NC machine tools. The two-step estimation method is used to estimate the parameters of baseline failure function and the coefficients of working conditions. The Bootstrap resamples are obtained by the Bootstrap resampling method. The parameters of baseline failure function and the coefficients of working conditions are estimated simultaneously by maximum likelihood estimation. The Power function is used to establish the change rate function of MTBF interval estimation. The result of the case study indicates that the testing periods under different covariates obtained by the proposed method are quite different from that obtained by the method without considering working conditions covariates. Therefore, the working conditions should not be ignored when the testing period is determined.

\section{Data Availability}

The data used to support the findings of this study are included within the article.

\section{Conflicts of Interest}

The authors declare that they have no conflicts of interest.

\section{Acknowledgments}

Research in this paper was supported by Jilin Provincial Education Department of Research on Reliability Modeling and Evaluation Technology of NC Equipment under Variable Environment or Conditions [Grant no. JJKH20180327KJ] and the Science and Technology Bureau of Jilin City [Grant no. 2013121010].

\section{References}

[1] H. Z. Li, Z. J. Yang, and B. B. Xu, "Design of testing period for reliability assessment of NC machine tools," Journal of Jilin University (Engineering and Technology), vol. 9, pp. 1521-1527, 2016.

[2] S. W. McKane, L. A. Escobar, and W. Q. Meeker, "Sample size and number of failure requirements for demonstration tests with log-location-scale distributions and failure censoring," Technometrics. A Journal of Statistics for the Physical, Chemical and Engineering Sciences, vol. 47, no. 2, pp. 182-190, 2005.

[3] J. He, H.-Z. Zhao, and Q. Fu, "Interval estimation and sample size calculation for ATR algorithm classification accuracy," Systems Engineering and Electronics, vol. 29, no. 7, pp. 1021-1026, 2007.

[4] H. R. Guo and R. Pan, "On determining sample size and testing duration of repairable system test," in Proceedings of the 2008 Annual Reliability and Maintainability Symposium, pp. 120-125, IEEE Computer Society, Washing DC, USA, 2008.

[5] H. Guo, Study on the intensifying factor of reliability test on automotive proving ground, Zhenjiang University, Hangzhou, China, 2003.

[6] W. H. Chen and H. S. Li, "Accelerated life test and statistical analysis of aerospace electrical connectors under multiple environment stresses," Journal of Zhenjiang University, vol. 40, no. 2, pp. 348-351, 2006.
[7] Q. N. Tan, X. Y. Wei, Z. W. Huang, and Y. F. Meng, "Reliablity evaluation method of electronic system under variable environments and working conditions," Journal of Central South University (Science and Technology), vol. 44, no. 8, pp. 32543260, 2013.

[8] J.-Y. Li, J.-X. Wang, G.-W. Zhou, W. Pu, and Z.-H. Wang, "Accelerated life testing of harmonic driver in space lubrication," Proceedings of the Institution of Mechanical Engineers, Part J: Journal of Engineering Tribology, vol. 229, no. 12, pp. 1491-1502, 2015.

[9] L. N. Ren, R. Z. Yuan, J. Liu, and C. L. Lei, "Whole lifecycle reliability assessment of multiple NC machine tools with minimal repair," Journal of Shanghai Jiao Tong University, vol. 49, no. 1, pp. 19-23, 2015.

[10] X. Z. Zhou, Research on Reliability Assessment Based on Bayesian Methods, Huazhong University of Science and Technology, Wuhan, China, 2007.

[11] H. Z. Li, Z. J. Yang, B. B. Xu, C. H. Chen, Y. N. Kan, and G. F. Liu, "Reliability evaluation of NC machine tools considering working condtions," in Mathematical Problems in Engineering, pp. 1-11, 2016.

[12] A. M. Zoubir and D. R. Iskandler, "Bootstrap methods and applications," IEEE Signal Processing Magazine, vol. 24, no. 4, pp. 10-19, 2007.

[13] Y. Han and L. C. Han, General Theory of Statistics, Beijing University Press, Beijing, China, 2012. 


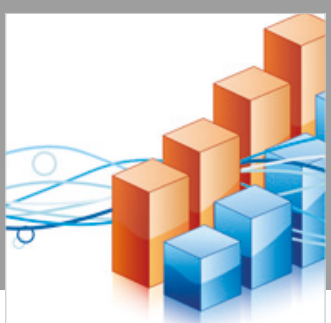

Advances in

Operations Research

\section{-n-m}
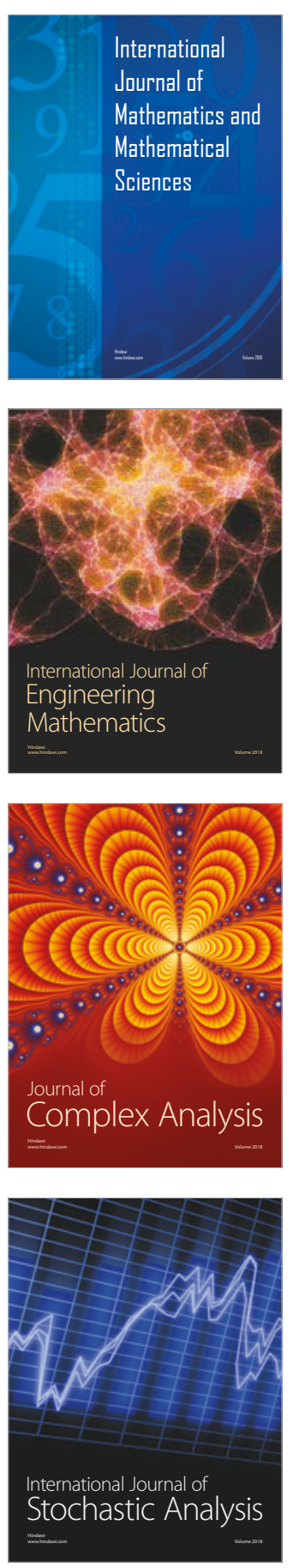
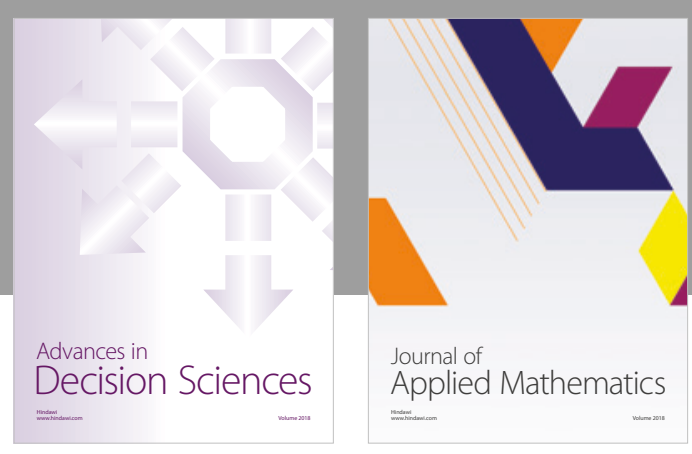

Journal of

Applied Mathematics
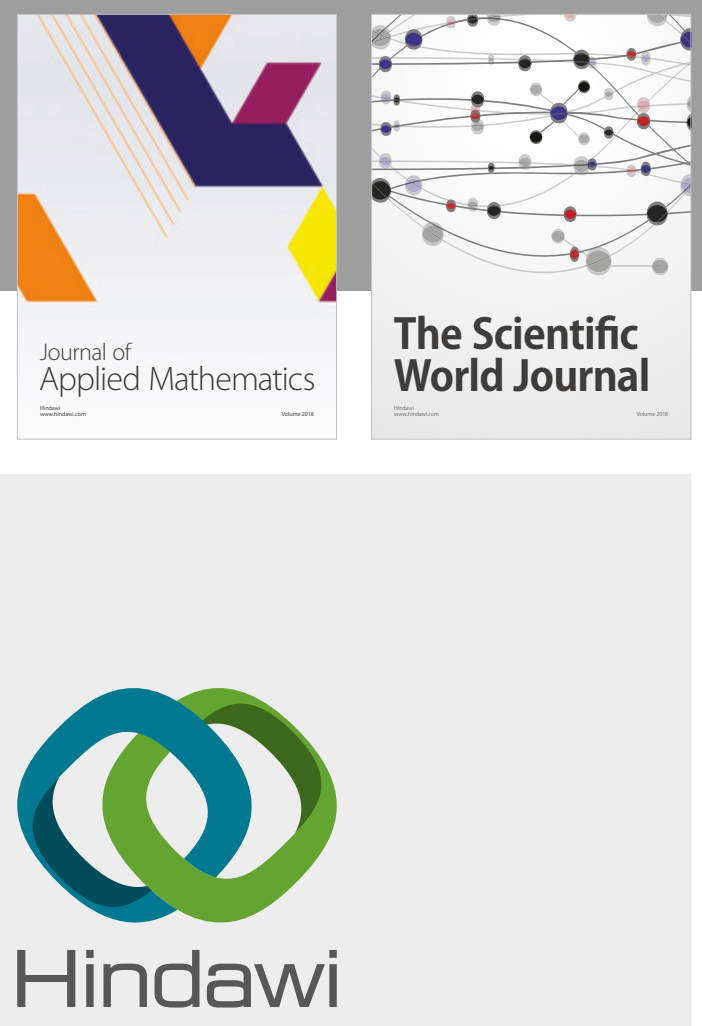

Submit your manuscripts at

www.hindawi.com

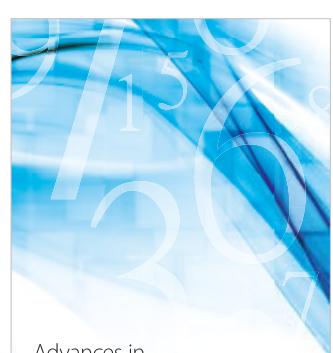

Advances in
Numerical Analysis
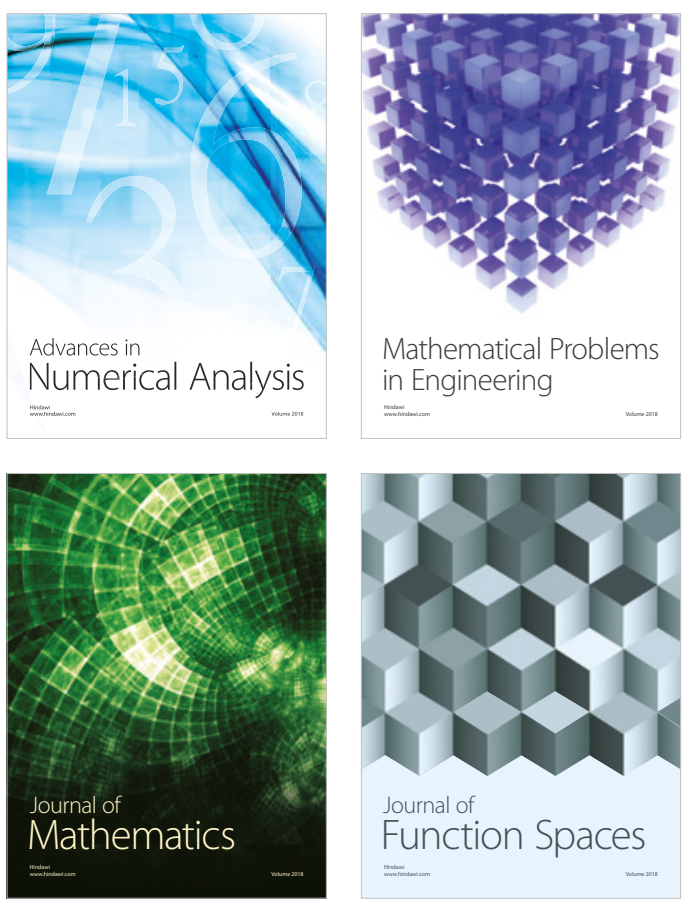

Mathematical Problems in Engineering

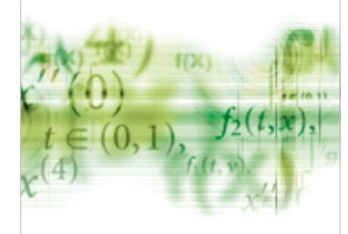

International Journal of

Differential Equations

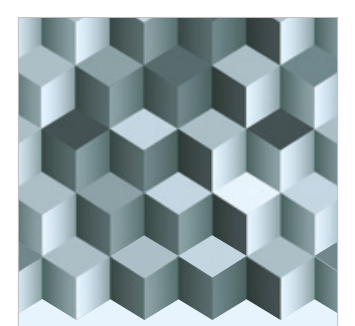

Journal of

Function Spaces

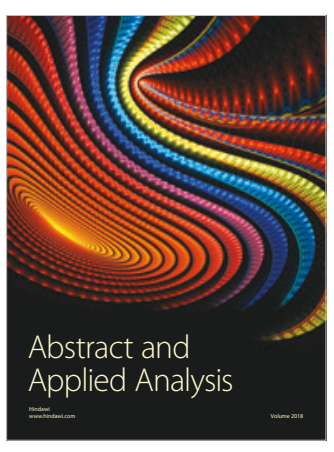

The Scientific

World Journal

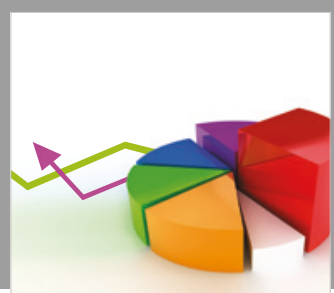

Journal of

Probability and Statistics
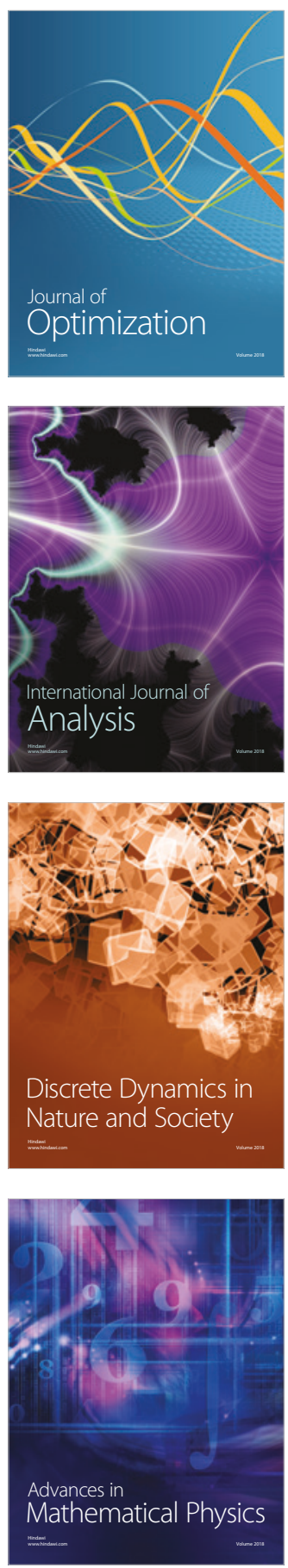